\title{
Joint Activity/Attenuation Reconstruction in SPECT Using Photopeak and Scatter Sinograms
}

\author{
Alexandre Bousse*, Avishay Sidlesky, Nathaniel Roth, Alaleh Rashidnasab, Kris Thielemans, Senior \\ Member, IEEE, Brian F. Hutton, Senior Member, IEEE
}

\begin{abstract}
This work presents the headlines of a joint activity/attenuation reconstruction in SPECT, based on the maximisation of the scatter and non-scatter data joint log-likelihood. The activity image is updated with standard expectation maximisation (EM) whereas the attenuation is updated with a quasiNewton line-search. Results on simulation demonstrates that the utilisation of scatter considerably reduces the ill-posedness of the intitial reconstruction problem with non-scatter counts only. Results on phantom data show that using scatter allows to achieve myocardium reconstruction similar to an EM reconstruction with CT attenuation correction.
\end{abstract}

Index Terms-SPECT, scatter, attenuation estimation, maximum-likelihood.

\section{INTRODUCTION}

$\mathbf{J}$ OINT reconstruction of the activity distribution and the attenuation map (JRAA) in single-photon emission computed tomography (SPECT) and positron emission tomography (PET) is a challenging field of research [1]-[3]. It is an ill-posed problem with infinitely many solutions which makes it difficult to obtain a reasonable solution without a priori knowledge on the attenuation map. Time-of-flight PET can greatly reduce the ill-posedness [3] but it only allows to recover the attenuation coefficient up to a constant [4].

The utilisation of scatter data can provide the missing information to estimate the attenuation map, as it turns the initial problem into a "2 equations-2 unknowns" system. Previous work from [5] showed promising results, but the utilisation of heuristics complicates the implementation. In this work we summarise a reconstruction scheme based on the maximisation of the joint likelihood of scatter and non-scatter data, treated as 2 independent random variables. The activity is updated with an expectation maximisation (EM) step [6] whereas the attenuation is updated with a Quasi-newton linesearch [7]. Our aim is also to investigate the ill-posed nature of JRAA from emission data, with and without scatter, and therefore no a priori information on the attenuation was used. Preliminary results on simulated and phantom data are shown in Section III.

This work was supported by the NIHR-funded UCH Biomedical Research Centre and Spectrum Dynamics. Asterisk indicates corresponding author.

${ }^{*}$ A. Bousse, A. Rashidnasab, K. Thielemans and B. F. Hutton are with the Institute of Nuclear Medicine, University College London, London NW1 2BU, UK (email: a.bousse@ucl.ac.uk).

A. Sidlesky and N. Roth are with Spectrum Dynamics, Caesarea 30889, Israel.

B. F. Hutton is also with the Centre for Medical Radiation Physics, University of Wollongong, Wollongong NSW 2522, Australia.

\section{METHOD}

\section{A. No Scatter Information}

SPECT image reconstruction consists of estimating a volumetric image $\boldsymbol{f}=\left(f_{1}, \ldots, f_{n_{\mathrm{v}}}\right)^{\top} \in \mathbb{R}_{+}^{n_{\mathrm{v}}}$ such that the expected counts $\overline{\boldsymbol{g}}(\boldsymbol{f}, \boldsymbol{\mu}) \in \mathbb{R}_{+}^{n_{\mathrm{d}}}$, defined as

$$
\bar{g}(\boldsymbol{f}, \boldsymbol{\mu})=\boldsymbol{P}(\boldsymbol{\mu}) \boldsymbol{f}+\boldsymbol{r},
$$

match the SPECT measurements $\boldsymbol{g} \in \mathbb{N}^{n_{\mathrm{d}}}$, where $\boldsymbol{r} \in \mathbb{R}_{+}^{n_{\mathrm{d}}}$ denotes the vector of expected scatter, $\boldsymbol{\mu}=\left(\mu_{1}, \ldots, \mu_{n_{\mathrm{v}}}\right)^{\top^{+}} \in$ $\mathbb{R}_{+}^{n_{\mathrm{v}}}$ is the attenuation map obtained from separate reconstruction such as X-ray computerised tomography (CT), $n_{\mathrm{v}}$ and $n_{\mathrm{d}}$ denote the number of voxels and detection bins respectively. The SPECT system matrix $\boldsymbol{P}(\boldsymbol{\mu}) \in \mathbb{R}_{+}^{n_{\mathrm{d}} \times n_{\mathrm{v}}}$ contains the probability that an emission occurring in voxel $j \in\left\{1, \ldots, n_{\mathrm{v}}\right\}$ is detected at bin $i \in\left\{1, \ldots, n_{\mathrm{d}}\right\}$ :

$$
[\boldsymbol{P}(\boldsymbol{\mu})]_{i, j}=p_{i, j} \exp \left(-\sum_{q=1}^{n_{\mathrm{v}}} \ell_{i, j ; q} \mu_{q}\right)
$$

where $\ell_{i, j ; q}$ is the fraction of the segment connecting $i$ and $j$ intersecting voxel $q$ and $p_{i, j}$ is the geometric contribution (i.e. the probability of detection in absence of attenuation medium). Reconstructing $f$ is traditionally achieved by maximisation of the likelihood [6], i.e. by solving

$$
\max _{\boldsymbol{f} \geq \mathbf{0}} L(\boldsymbol{g} \mid \overline{\boldsymbol{g}}(\boldsymbol{f}, \boldsymbol{\mu}))
$$

where $L(\boldsymbol{g} \mid \overline{\boldsymbol{g}}) \triangleq \sum_{i=1}^{n_{\mathrm{d}}} g_{i} \log \bar{g}_{i}-\bar{g}_{i}$ is the Poisson loglikelihood of the observable $\boldsymbol{g}$ conditionally to $\overline{\boldsymbol{g}}$.

In absence of an attenuation map, one could solve (3) jointly for $\boldsymbol{f}$ and $\boldsymbol{g}$, which is an approach namely referred to as maximum-likelihood reconstruction of attenuation and activity (MLAA) [2], and thus avoid CT measurement. Unfortunately, it is a very ill-posed reconstruction problem in absence of prior knowledge on $\boldsymbol{\mu}$,

\section{B. Separating Scatter and Photopeak Data}

1) Statistical Model: One option to reduce the ill-posedness of JRAA (or MLAA) is to separate scatter from the photopeak data in $\boldsymbol{g}$, and introduce a probabilistic model of the scatter events. This approach was introduced in [5]. We propose a similar reconstruction scheme based on the joint maximum likelihood of the scatter and photopeak events. We assume that the observed counts $\boldsymbol{g}$ is a sum of two independent Poisson processes,

$$
\boldsymbol{g}=\boldsymbol{g}^{\mathrm{pp}}+\boldsymbol{g}^{\mathrm{sc}}
$$


where $\boldsymbol{g}^{\mathrm{pp}}$ and $\boldsymbol{g}^{\mathrm{sc}}$ are the photopeak (unscattered) and scattered detections respectively. We also assume that $\boldsymbol{g}^{\mathrm{pp}}$ and $\boldsymbol{g}^{\mathrm{sc}}$ can be measured separately, and that $\boldsymbol{g}^{\mathrm{sc}}$ contains single scatter events only. The joint log-likelihood of $\left(\boldsymbol{g}^{\mathrm{pp}}, \boldsymbol{g}^{\mathrm{sc}}\right)$, conditionally to $(\boldsymbol{f}, \boldsymbol{\mu})$, is

$$
\Phi(\boldsymbol{f}, \boldsymbol{\mu}) \triangleq L\left(\boldsymbol{g}^{\mathrm{pp}} \mid \overline{\boldsymbol{g}}^{\mathrm{pp}}(\boldsymbol{f}, \boldsymbol{\mu})\right)+L\left(\boldsymbol{g}^{\mathrm{sc}} \mid \overline{\boldsymbol{g}}^{\mathrm{sc}}(\boldsymbol{f}, \boldsymbol{\mu})\right)
$$

where $\overline{\boldsymbol{g}}^{\mathrm{pp}}(\boldsymbol{f}, \boldsymbol{\mu}) \triangleq \boldsymbol{P}(\boldsymbol{\mu}) \boldsymbol{f}$ is the expected photopeak counts vector and $\overline{\boldsymbol{g}}^{\mathrm{sc}}(\boldsymbol{f}, \boldsymbol{\mu})$ is the expected scatter counts vector, which replaces $\boldsymbol{r}$ in (1). $\overline{\boldsymbol{g}}^{\mathrm{sc}}(\boldsymbol{f}, \boldsymbol{\mu})$ is defined at each bin $i \in\left\{1, \ldots, n_{\mathrm{d}}\right\}$ as

$$
\bar{g}_{i}^{\mathrm{sc}}(\boldsymbol{f}, \boldsymbol{\mu})=\sum_{k=1}^{n_{\mathrm{v}}} p_{i, k} \exp \left(-\sum_{q=1}^{n_{\mathrm{v}}} \ell_{i, k ; q} \mu_{q}\right) \lambda_{i, k}^{\mathrm{sc}}(\boldsymbol{f}, \boldsymbol{\mu}),
$$

where $\lambda_{i, k}^{\mathrm{sc}}(\boldsymbol{f}, \boldsymbol{\mu})$ is the number of photons scattered in $k$ towards $i$,

$\lambda_{i, k}^{\mathrm{sc}}(\boldsymbol{f}, \boldsymbol{\mu})=\left(1-\mathrm{e}^{-\mu_{k}}\right) \sum_{b=1}^{n_{\mathrm{v}}} f_{b} \omega_{k, b} \kappa_{k, b}^{i} \exp \left(-\sum_{p \neq k}^{n_{\mathrm{v}}} \ell_{k, b ; p} \mu_{p}\right)$,

$\kappa_{k, b}^{i}$ is the Klein-Nishina probability that a photon emitted from $b$, scattered in $k$, travels toward $i$, and $\omega_{k, b}$ is the fractional solid angle between $b$ and $k$. It is somehow worth noting that (5) corresponds to a projection of the "scatter activity volume" $\boldsymbol{\lambda}_{i}^{\mathrm{sc}} \triangleq\left(\lambda_{i, k}^{\mathrm{sc}}(\boldsymbol{f}, \boldsymbol{\mu})\right)_{k=1}^{n_{\mathrm{v}}} \in \mathbb{R}_{+}^{n_{\mathrm{v}}}$ with the SPECT system matrix $\boldsymbol{P}(\boldsymbol{\mu})$ defined in (2).

2) Optimisation: We propose an algorithm to "pseudo maximise" the joint log-likelihood $\Phi(\boldsymbol{f}, \boldsymbol{\mu})$, by alternating maximisation in $\boldsymbol{f}$ and $\boldsymbol{\mu}$.

The attenuation map $\boldsymbol{\mu}$ is updated with a line-search step on a quasi-Newton direction:

$$
\begin{aligned}
& \boldsymbol{\mu} \leftarrow \boldsymbol{\mu}-\alpha^{\star} \boldsymbol{B} \nabla_{\boldsymbol{\mu}} \Phi(\boldsymbol{f}, \boldsymbol{\mu}), \\
& \text { with } \quad \alpha^{\star}=\underset{\alpha \geq 0}{\arg \max } \Phi\left(\boldsymbol{f}, \boldsymbol{\mu}-\alpha \boldsymbol{B} \nabla_{\boldsymbol{\mu}} \Phi(\boldsymbol{f}, \boldsymbol{\mu})\right)
\end{aligned}
$$

where $\boldsymbol{B}$ is a negative-definite L-BFGS approximation of the inverse Hessian matrix of $\Phi$ in $\boldsymbol{\mu}$, computed following the implementation from [7]. The gradient $\nabla_{\boldsymbol{\mu}} \Phi(\boldsymbol{f}, \boldsymbol{\mu})$ was derived analytically, with GPU accelerated CUDA ${ }^{\circledR}$ implementation of the scatter likelihood gradient.

The activity image is updated with an EM update [6], using the photopeak data $\boldsymbol{g}^{\mathrm{pp}}$ and the SPECT system matrix $\boldsymbol{P}(\boldsymbol{\mu})$ (2). Scatter data $\boldsymbol{g}^{\mathrm{sc}}$ are ignored in this step. In that sense, because only $L\left(\boldsymbol{g}^{\mathrm{pp}} \mid \overline{\boldsymbol{g}}^{\mathrm{pp}}(\boldsymbol{f}, \boldsymbol{\mu})\right)$ is maximised in $\boldsymbol{f}$, the overall algorithm is not an "exact maximisation" of $\Phi(\boldsymbol{f}, \boldsymbol{\mu})$ as defined in (4).

\section{RESULTS}

\section{A. Simulated Data}

We created $64 \times 64 \times 64$ attenuation and activity volumes (Fig. 1(a) and 1(b)), denoted $\boldsymbol{\mu}^{\star}$ and $\boldsymbol{f}^{\star}$ respectively. Noiseless photopeak and scatter sinograms $\boldsymbol{g}^{\mathrm{pp}}=\overline{\boldsymbol{g}}^{\mathrm{pp}}\left(\boldsymbol{f}^{\star}, \boldsymbol{\mu}^{\star}\right)$ and $\boldsymbol{g}^{\mathrm{sc}}=\overline{\boldsymbol{g}}^{\mathrm{sc}}\left(\boldsymbol{f}^{\star}, \boldsymbol{\mu}^{\star}\right)$ were generated as input data for reconstruction (which implies that $\left(\boldsymbol{f}^{\star}, \boldsymbol{\mu}^{\star}\right)$ is a maximiser for $\Phi$ ). The $\mu$-values and Klein-Nishina factors we used correspond to ${ }^{99} \mathrm{Tc}$ emissions.
We performed 4 reconstructions: (i) EM activity reconstruction from $\boldsymbol{g}^{\text {pp }}$ using the true attenuation map $\boldsymbol{\mu}^{\star}$ (EM-AC), Fig. 1(c); (ii) EM activity reconstruction from $\boldsymbol{g}^{\mathrm{pp}}$ without attenuation correction (EM-noAC), Fig. 1(f); (iii) JRAA reconstruction of the attenuation from $\boldsymbol{g}^{\mathrm{pp}}$ by maximisation of $(\boldsymbol{f}, \boldsymbol{\mu}) \mapsto L\left(\boldsymbol{g}^{\mathrm{pp}} \mid \overline{\boldsymbol{g}}^{\mathrm{pp}}(\boldsymbol{f}, \boldsymbol{\mu})\right)$ using L-BFGS quasiNewton optimisation in $\boldsymbol{\mu}$ (JRM-pp), Fig. 1(d), and EM for the activity, Fig. 1(e) (we did not use the MLAA algorithm with prior proposed in [2]); (iv) proposed approach: JRAA of the attenuation, Fig. 1(g), and the activity, Fig. 1(h), by maximisation of the joint-likelihood $\Phi$, using photopeak and scatter data (JRAA-sc).

In absence of Poisson noise, EM-AC and EM-noAC (Fig. 1(c) and 1(f)) are respectively the "best and worst" results that can be achieved. As no prior information on $\boldsymbol{\mu}$ was used, JRAA-pp heavily suffers from the non-uniqueness of a solution. We indeed observe pronounced cross-talk in the myocardium region resulting in underestimated attenuation and activity, as also observed in [2]. In addition, the lungs are not visible in both the activity and attenuation. JRAAsc, which uses both $\boldsymbol{g}^{\mathrm{pp}}$ and $\boldsymbol{g}^{\mathrm{sc}}$, provides significantly better results: the lungs are visible (in the activity and attenuation images) as well as the ribs, and the myocardium activity is better reconstructed. Cross-talk in the myocardium is still present but is greatly reduced as compared to JRAA-pp. The cross-section profile curves (Figure 2) show that JRAA-sc sits between JRAA-pp EM-AC.

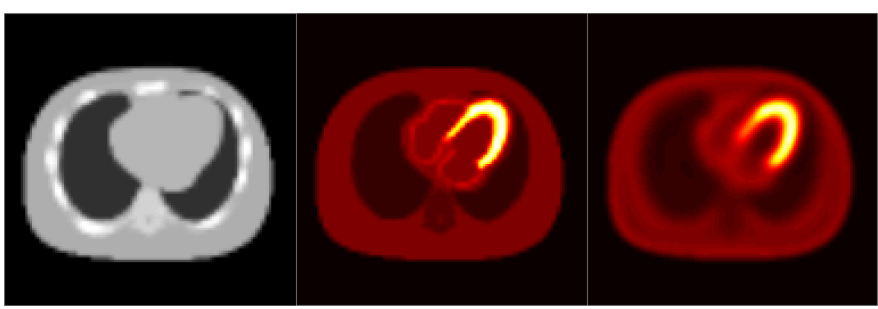

(a)

(b)

(c)

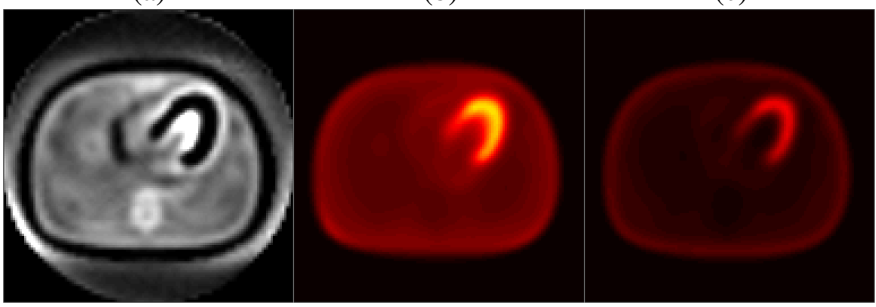

(d)

(e)

(f)

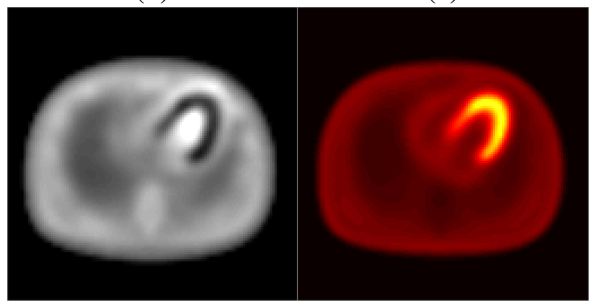

$(\mathrm{g})$

(h)

Fig. 1: (a) Attenuation map phantom; (b) activity phantom (c) EM-AC reconstructed activity; JRAA-pp reconstructed (d) attenuation and (e) activity; (f) EM-noAC reconstructed activity; JRAA-sc reconstructed (g) attenuation and (h) activity. 


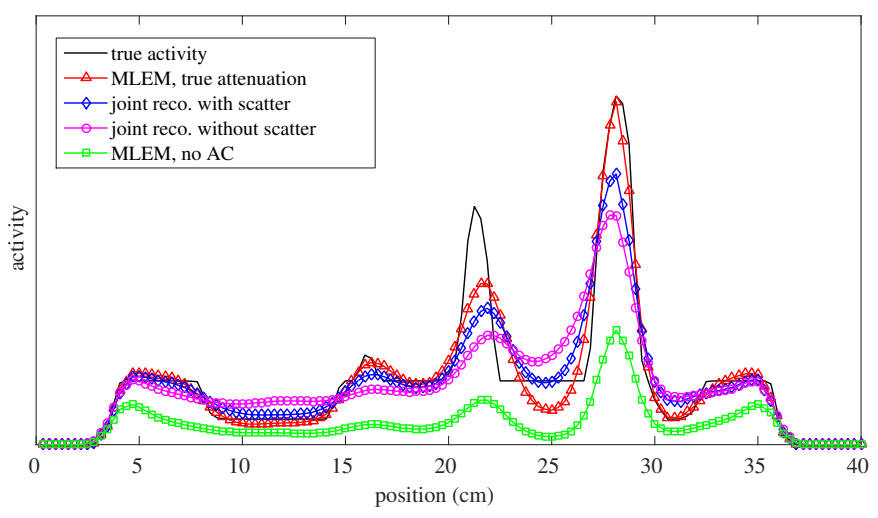

Fig. 2: Activity profiles.

\section{B. Real Phantom SPECT Acquisition}

An anthropomorphic torso phantom (Fig. 3) acquisition, with a GE Discovery 670 (LEHR), was performed at the Sheba Medical Center, Tel Hashomer, Israel. Photopeak and scatter energy windows were respectively set to $126-154 \mathrm{KeV}$ and $100-126 \mathrm{KeV}$. No scatter correction were applied to the photopeak data and we did not subtract non-scatter detections from the scatter window data.

We proceeded with 3 reconstructions: EM reconstruction with CT attenuation correction (EM-CT-AC, see Fig. 4(a) and 5(a) for the activity and attenuation respectively), EM without attenuation correction (EM-noAC, Fig. 4(b)), and the proposed JRAA-sc (see Fig. 4(c) and 5(b) for the activity and attenuation respectively). Due to the ill-posed nature of the problem, we implemented some further constraints, such as limiting the range of values of the reconstructed attenuation coefficients. The EM-noAC image was rescaled for display purpose.

The JRAA-sc reconstructed activity suffers from crosstalk near the myocardium, and the lungs are poorly estimated, as observed with simulated data. However, the contrast between the myocardium and the soft tissues is more similar to EM-CT$\mathrm{AC}$, whereas the soft tissues in the EM-noAC reconstruction appear over estimated. The JRAA-sc estimated attenuation fails to estimate the lungs, but the boundaries well defined and the spine was correctly reconstructed.

The myocardium reconstruction quality can be assessed with a bullseye representation (Fig. 6). The EM-noAC bullseye (Fig. 6(b)) suffers from severe defects that are significantly reduced when using JRAA-sc (Fig. 6(c)). This suggest that our approach using scatter for attenuation correction potentially achieves correct estimation of the myocardium activity distribution.

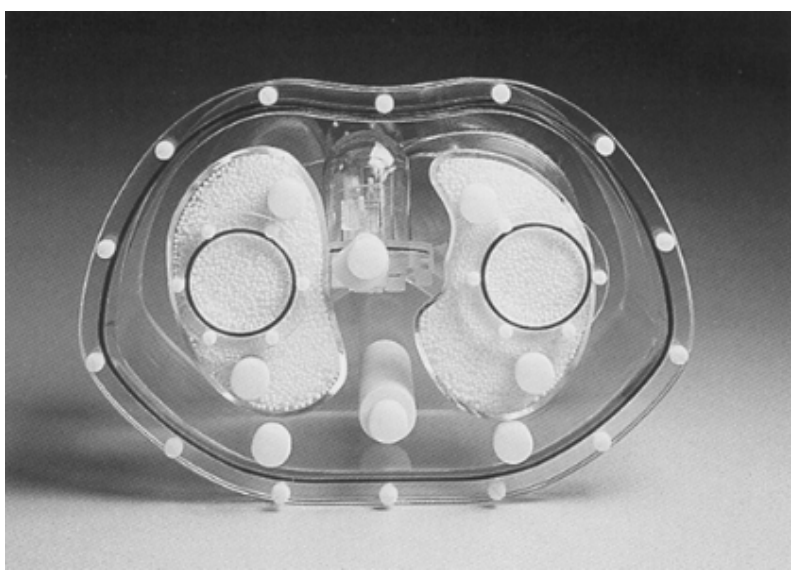

Fig. 3: Torso phantom used for SPECT acquisition.

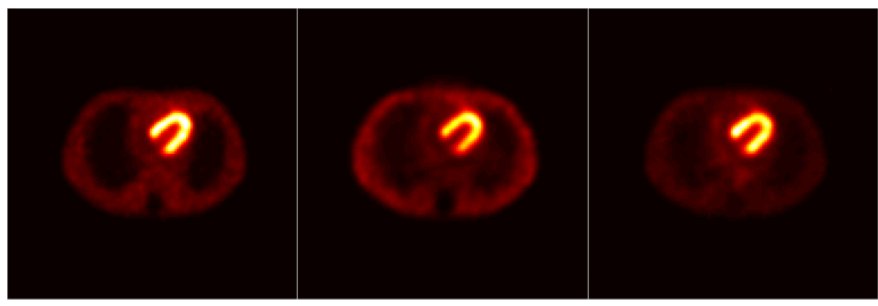

(a)

(b)

(c)

Fig. 4: Activity images: (a) EM-CT-AC; (b), EM-noAC; (c) JRAA-sc.

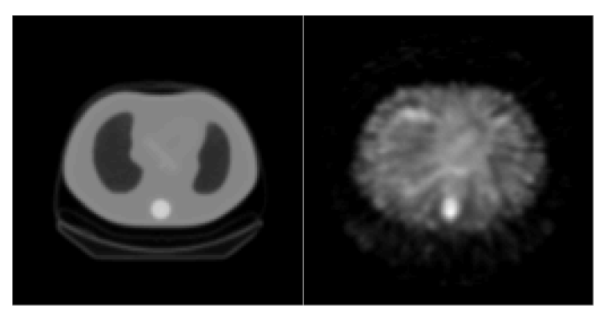

(a)

(b)

Fig. 5: (a) CT-derived attenuation map; (b) Estimated attenuation using JRAAsc.

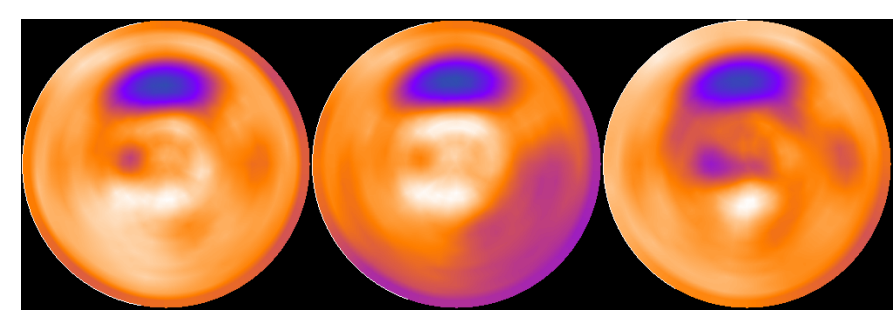

(a)

(b)

(c)

Fig. 6: Bullseye representation of the myocardium: (a) EM-CT-AC; (b) EMnoAC; (c) JRAA-sc.

\section{CONCLUSION}

Using scatter in addition to photopeak information for JRAA does, as expected, reduce the ill-posedness of the problem. However, crosstalk is still present, especially in the myocardium area. Further efforts should be made to reduce crosstalk, for example by incorporating prior information 
on the attenuation map. Real data experiment showed that the myocardium can be accurately reconstructed with JRAA with scatter. The reconstruction of the attenuation map from photopeak and scatter windows is challenging, but can be improved using priors and also by correcting for scatter in the photopeak window and subtracting non-scatter counts from the scatter window.

\section{ACKNOWLEDGMENT}

Sheba Medical Center, Tel Hashomer, Israel, for providing real phantom data.

\section{REFERENCES}

[1] Y. Censor, D. E. Gustafson et al., "A new approach to the emission computerized tomography problem: Simultaneous calculation of attenuation and activity coefficient," IEEE Trans. Nucl. Sci., vol. 26, no. 2, pp. 27752779,1979

[2] J. Nuyts, P. Dupont et al., "Simultaneous maximum A Posteriori reconstruction of attenuation and activity distributions from emission sinograms," IEEE Trans. Med. Imag., vol. 18, no. 5, pp. 393-403, 1999.

[3] A. Rezaei, M. Defrise et al., "Simultaneous reconstruction of activity and attenuation in time-of-flight PET," IEEE Trans. Med. Imag., vol. 31, no. 12 , pp. 2224-2233, 2012.

[4] M. Defrise, A. Rezaei, and J. Nuyts, "Time-of-flight PET data determine the attenuation sinogram up to a constant," Phys. Med. Biol., vol. 57, pp. 885-899, 2012.

[5] S. C. Cade, S. Arridge et al., "Use of measured scatter data for the attenuation correction of single photon emission tomography without transmission scanning," Med. Phys., vol. 40, no. 8, 2013.

[6] L. A. Shepp and Y. Vardi, "Maximum likelihood reconstruction for emission tomography," IEEE Trans. Med. Imag., vol. 1, no. 2, pp. 113$122,1982$.

[7] R. H. Byrd, P. Lu, and J. Nocedal, "A limited memory algorithm for bound constrained optimization," SIAM J. Sci. Stat. Comp., vol. 16, no. 5, pp. 1190-1208, 1995. 